\section{Capilaroscopia da Prega Ungueal em Crianças Com Diabetes Mellitus Tipo I}

\section{RESUMO}

O estudo teve como objetivo identificar o aspecto morfológico da microcirculação, em crianças com e sem diabetes mellitus (DM), pela capilaroscopia de prega ungueal das mãos. Foram selecionadas 30 crianças com DM do tipo 1 (DM1) e 24 crianças sem DM, pareadas quanto ao sexo, cor e idade. Encontrou-se aumentos significativos de freqüência de dilatação e tortuosidade de ramos transverso e eferente e microectasias em relação aos controles. Não houve aumento significativo da freqüência de edema nas crianças com DM pela alta freqüência desta alteração no grupo controle. Ao contrário dos adultos com DM, houve predominância de espasticidade do ramo aferente e o longo comprimento das alças foi preservado. O padrão descrito na microcirculação no presente estudo pode ser importante para o diagnóstico das complicações vasculares da doença. (Arq Bras Endocrinol Metab 2001;45/5:441-446)

Unitermos: Diabetes mellitus tipo 1; Microcirculação; Capilaroscopia; Microangiopatia.

\begin{abstract}
Thirty patients with type 1 diabetes (DM1) (target group) and 24 controls were studied through nailfold capillaroscopy. Both groups were age, color and sex matched. Statistically significant high frequencies of edema, dilatation and tortuosity of efferent branches and microectasy were found in microcirculation. Dermal papillae rectification as an isolated finding did not characterize edema because its frequency was high among controls. Rather than is reported in type 2 DM, afferent branch spasticity and long loops were significantly frequent among the target group. These data suggest that nailfold capillaroscopy may be an important method for the diagnosis and follow up of vascular abnormalities in DM1. (Arq Bras Endocrinol Metab 2001;45/5:441-446)
\end{abstract}

Keywords: Diabetes mellitus type 1; Microcirculation; Capillaroscopy; Microangiopathy.

A Descoberta Da insulina, EM 1922, aumentou a longevidade dos pacientes com diabetes (DM) pela melhoria do controle metabólico (2). As complicações vasculares, no entanto, passaram a ganhar importância pelo comprometimento da qualidade de vida, redução da capacidade produtiva e aumento da mortalidade de pacientes com DM. Desde então, as alterações da microcirculação vêm sendo estudadas com mais profundidade.

Jager, em 1855, e mais tarde Weiss, em 1916, já haviam observado padrão vascular anormal no fundo de olho de diabéticos, que foi claramente relacionado à doença por Waite e Beetham, em 1935, e descrito no glomérulo por Kimmelstiel e Wilson um ano depois $(23,25,44,45)$.

O termo microangiopatia diabética foi criado por Lundbaeck em 1953, que descreveu lesões anátomo-patológicas caracterizadas, inicial-

\section{artigo original}

\author{
Therezinba J. Fernandes \\ Edda M.T. Bernardini \\ Iara C. Morais \\ Claudia Castilho \\ Vera Lucia R.C. Halfoun
}

\author{
Departamento de Clínica Médica \\ (TJF, EMTB, ICM, VLRCH) e \\ Departamento de Pediatria (CC), \\ Faculdade de Medicina e Serviço de \\ Angiologia, Hospital Universitário \\ Clementino Fraga Filho, \\ Universidade Federal do Rio de Janeiro- \\ UFRJ, Rio de Janeiro, RJ.
}

Recebido em 27/04/00 Revisado em 14/03/01 Aceito em 23/03/01 
mente, por espessamento da membrana basal, quando corada pelo PAS e vista à microscopia óptica. Estas alterações foram, posteriormente, confirmadas à microscopia eletrônica por Farquhar $(20,29)$.

A noção de que a microangiopatia diabética é um processo dinâmico, provavelmente desencadeado por alterações da permeabilidade capilar, foi estabelecida por Ditzel, em 1967, e desenvolvida posteriormente por vários autores $(12,20,44,45)$.

Após a introdução da angiografia fluoresceínica e da videocapilaroscopia, tem sido possível documentar, através da imagem, as diversas etapas de alteração de forma da alça capilar em vários tecidos ou órgãos, tanto em animais quanto em humanos $(6,7,13,36,46)$.

$\mathrm{Na}$ capilaroscopia da prega ungueal, vários autores observaram um padrão de visibilidade difícil pela presença de edema, dilatação e tortuosidade de ramos eferentes, encurtamento de alças e presença de microectasias no DM2 e em parentes $(4,10,11,14,22,33,34)$.

Mayer (1921) demonstrou que, na criança normal, o aspecto da microcirculação observado na capilaroscopia modificava-se nas diversas etapas do desenvolvimento infantil (30). Outros autores, no entanto, só encontraram alterações diferentes do adulto nos primeiros meses de vida $(8,19)$.

$\mathrm{Na}$ criança com DM há poucos estudos publicados, sobretudo em nosso meio $(8-10,25,35,37,41)$. A presença de microectasias, tortuosidade e de dilatação do ramo eferente tem sido um achado comum $(11,25,35,37)$. Há polêmica, no entanto, sobre a existência de espasticidade, edema, hemorragia e neovascularização $(11,41)$.

O objetivo deste trabalho é identificar as alterações da microcirculação da prega ungueal, através da capilaroscopia, em crianças com DMl, comparadas às normais.

\section{CASUÍSTICA}

Foram estudadas 30 crianças com DMl (grupo alvo), cujas idades variaram de 53 a 155 meses $(114,2 \pm 25,7$ meses), selecionadas no ambulatório do Instituto de
Puericultura e Pediatria Martagão Gesteira da Universidade Federal do Rio de Janeiro (UFRJ). Destas, 17 tinham menos de 5 anos de duração da doença e sete entre 5 e 9 anos. Todas tinham fundo de olho normal e graus variáveis de compensação metabólica.

O grupo controle foi formado por 24 crianças sem DM e sem história familiar da doença, selecionadas entre parentes de funcionários do Hospital Universitário da UFRJ, pareadas quanto à idade $(\mathrm{p}>$ $0,05)$, cor e sexo (tabela 1 ).

\section{MÉTODOS}

A capilaroscopia foi feita com estereoscópio Wild M3C, com aumentos de 6,4 a 10 vezes, iluminado por feixe de fibra óptica a 45 graus com lâmpada de halogênio de $6 \mathrm{~V} / 10 \mathrm{~W}$.

Os exames foram realizados nos $2^{\text {os }}, 3^{\text {os }}$ e $4^{\text {os }}$ dedos das mãos, em sala com iluminação fria, sem ruído e com temperatura ambiente constante, após aplicação de uma gota de óleo de cedro nas pregas ungueais.

A capilaroscopia era realizada simultaneamente por dois observadores, cuja concordância havia sido previamente estabelecida ( $k>60)$, sendo, os dados obtidos, registrados em ficha padrão. Em caso de dúvida um terceiro examinador era convidado a realizar o exame.

Foram considerados os seguintes achados:

- visibilidade: normal ou difícil, no caso de não obtenção de foco nas alças;

- edema: presente quando havia retificação da papila associada à presença de névoa translúcida em todo o campo e/ou verticalização de alças e dificuldade de foco;

- forma das alças: em grampo ou tortuosas;

- $\quad$ ramos capilares: espásticos, normais ou dilatados em relação a cada segmento da alça (aferente, transverso ou eferente). A modificação da relação arteríolo-venular determinou o grau de espasticidade ou vasodilatação;

- microectasias: formações saculares localizadas em qualquer local da alça.

Tabela 1. Características da amostra: sexo e cor.

\begin{tabular}{lcrrc}
\hline Grupos / Variáveis & \multicolumn{2}{c}{ Sexo } & \multicolumn{2}{c}{ Cor } \\
\hline & MASCULINO & FEMININO & BRANCA & NÃO BRANCA \\
\hline Alvo & $17(56,7 \%)$ & $13(43,3 \%)$ & $17(56,7 \%)$ & $13(43,3 \%)$ \\
Controle & $14(58,3 \%)$ & $10(41,7 \%)$ & $16(66,7 \%)$ & $8(33,3 \%)$ \\
\hline \multicolumn{4}{l}{$\chi^{2}=0 ; p=1 ;$ Fischer $=0,562$} & $\chi^{2}=0,219 ; p=0,6 ;$ Fischer $=0,321$
\end{tabular}


Tabela 2. Resumo dos achados capilaroscópicos.

\begin{tabular}{|c|c|c|c|c|c|c|c|c|}
\hline \multirow{3}{*}{$\begin{array}{l}\text { Grupos } \\
\text { Variáveis } \\
\text { Encurtamento de alças } \\
p=0,0588\end{array}$} & \multicolumn{4}{|c|}{ Controle } & \multicolumn{4}{|c|}{ Alvo } \\
\hline & \multicolumn{2}{|c|}{ presente } & \multicolumn{2}{|c|}{ ausente } & \multicolumn{2}{|c|}{ presente } & \multicolumn{2}{|c|}{ ausente } \\
\hline & 7 & $29,2 \%$ & 17 & $70,8 \%$ & 12 & $40 \%$ & 18 & $60 \%$ \\
\hline $\begin{array}{l}\text { Tortuosidade capilar } \\
p<0,001\end{array}$ & 1 & $4,2 \%$ & 23 & $95, \%$ & 15 & $50 \%$ & 15 & $50 \%$ \\
\hline $\begin{array}{l}\text { Edema Intersticial } \\
p=0,154\end{array}$ & 16 & $66,6 \%$ & 8 & $33,7 \%$ & 26 & $86,7 \%$ & 4 & $13,3 \%$ \\
\hline $\begin{array}{l}\text { Microectasias } \\
p<0,001\end{array}$ & 0 & $0 \%$ & 24 & $100 \%$ & 24 & $80 \%$ & 6 & $20 \%$ \\
\hline $\begin{array}{l}\text { Espasticidade aferente * } \\
p<0,001\end{array}$ & 0 & $0 \%$ & 23 & $100 \%$ & 10 & $34,4 \%$ & 19 & $65,6 \%$ \\
\hline $\begin{array}{l}\text { Dilatação transverso } \\
p<0,016\end{array}$ & 2 & $8,3 \%$ & 22 & $91,7 \%$ & 11 & $42,34 \%$ & 15 & $57,7 \%$ \\
\hline $\begin{array}{l}\text { Dilatação eferente } \\
\mathrm{p}<0,001\end{array}$ & 2 & $8,3 \%$ & 22 & $91,7 \%$ & 14 & $53,8 \%$ & 12 & $46,2 \%$ \\
\hline
\end{tabular}

* o tamanho amostral foi reduzido pela falta de concordância entre examinadores para a determinada variável ou dificuldade de visualização de segmentos pela presença de edema ou má visibilidade.

Os resultados foram tabulados e comparados em tabelas 2:2 e tratados pelos métodos do quiquadrado ou teste de Fischer, com nível de significância de $0,05 \%$.

\section{RESULTADOS}

As presenças de: microectasia, tortuosidade de alças, espasticidade de ramo aferente e dilatação dos segmentos transverso e eferente foram significativamente mais freqüiente nos diabéticos que nos controles $(\mathrm{p}<0,01$ exceto para dilatação do transverso, onde $\mathrm{p}<0,05)$. Não houve diferença significativa na freqüência de alças encurtadas e edema em relação aos controles (tabela 2).

Embora a visibilidade dos campos fosse melhor no grupo controle, havia retificação das papilas dérmicas, aumentando o percentual de casos de edema $(66,7)$; no grupo alvo, a retificação de papila associada ao aspecto nebuloso do campo quase sempre prejudicou a visibilidade do campo $(87,6 \%)$ (tabela 2$)$.

\section{DISCUSSÃO}

A literatura médica apresenta poucos trabalhos sobre o estudo dos microvasos da prega ungueal, tanto na criança sadia quanto naquela com DM. No adulto, entretanto, ela é rica no que se refere ao estudo da micro- circulação. Nos últimos anos, as pesquisas, através da capilaroscopia, têm crescido em importância como método de diagnóstico, apoiadas nos avanços dos conhecimentos fisiopatológicos.

Os achados visibilidade difícil, de edema, de dilatação do ramo eferente e de microectasia têm sido encontrados com freqüência no DM2 (4-7).

Bollinger e cols. (7), em 1979, ao estudarem a prega ungueal de indivíduos normais usando a fluoresceína sódica, observaram as trocas transcapilares acompanhando o movimento das partículas, documentando a sua saída e sua distribuição em áreas pericapilares, tanto no halo quanto nas partes mais remotas. Nos com Dm, o contraste apareceu exibindo imagem borrada e leitosa dos capilares, e se difundiu mais rapidamente que nos controles. Nestes, o halo pericapilar permaneceu por até 40 a 50 minutos, ao contrário dos com DM, onde a difusão para áreas mais remotas foi muito mais rápida. A remoção do contraste nestas áreas foi mais lenta que nos controles. Estes achados demonstraram o aumento de permeabilidade capilar naqueles com DM, justificando a dificuldade de visualização de alças na capilaroscopia e o edema.

Em nosso estudo, a capilaroscopia realizada em crianças com DM, entre 4 e 12 anos de idade, evidenciou a perda da nitidez das alças capilares com má visibilidade do campo e a retificação das papilas, carac- 
terizando o edema em 26 (86,7\%) (tabela 2). No grupo controle, $16(66,7 \%)$ crianças tinham edema caracterizado por retificação de papilas e apenas 7 $(29,17 \%)$ mostraram visibilidade difícil.

Acreditamos que o edema discreto, encontrado em ambos os grupos, tenha ocorrido pela forma de socialização infantil nos trópicos que, proporcionando maior liberdade fora do lar e facilitando a atividade recreativa e esportiva, poderia torná-las mais vulneráveis aos microtraumas nas mãos. Além disso, a onicofagia (Cony e cols., em 1992) pode ser um fator importante na gênese deste tipo de edema (10). Por outro lado, a maior temperatura ambiente facilita a vasodilatação crônica e, conseqüentemente, o edema. Deve-se levar em consideração, também, o possível contato com substâncias agressivas ao tecido (Bernardini, 1992) (4).

Segundo Bollinger e cols. (1982), o edema faz surgir, no DM, mancha de aspecto leitoso que borra os ramos capilares, aparentando um encurtamento da alça, que prejudica a visibilidade $(5,6)$. Entretanto, não encontramos encurtamento de alças na maioria das crianças com DM estudadas. Este fato pode ser explicado pela melhor transparência da pele nesta faixa etária o que permite, ao contrário do adulto, uma melhor visibilidade de alças (2l).

No DM, o aumento de permeabilidade foi associado à dilatação de alça venular por vários autores $(13,31,43,47)$.

Estudando vasos da retina e da pele, em 1968, Ditzel observou que, em situações de mau controle da doença, a circulação local respondia com vasodilatação ao nível venular, concluindo que ela seria produzida por modificações metabólicas tissulares que acarretariam estase venosa (13).

Outros autores encontraram dilatação do ramo eferente do capilar em pacientes com DM e esta foi associada à perda do tônus de parede do vaso (4-7,11-18).

Nas crianças examinadas encontramos prevalência de dilatação dos ramos eferentes $(\mathrm{p}<0,001)$ e do segmento transverso $(\mathrm{p}=0,016)$ (tabela 2$)$. Este último foi descrito por Landau \& Davis (1960) e por Kiesewetter e cols. (1986) que, entretanto, não a reconheceram como lesão característica do $\operatorname{DM}(25,27)$.

Em nossa observação, o ramo aferente do capilar apresentou-se espástico em $34,48 \%$ das crianças do grupo alvo $(\mathrm{p}<0,001)$. Isto foi explicado por Ditzel, em 1968, como uma constrição do ramo arterial que ocorria no momento da anóxia do tecido, fato este confirmado por McMillan (1975) (13,31).

Kiesewetter e cols. (1986) encontraram tortuosidade da alça capilar em crianças com DM e justificaram essa alteração como um efeito da diminuição das células de sustentação com conseqüente perda do tônus da parede do microvaso. Estas alterações seriam um dos fatores desencadeantes da diminuição da velocidade do eritrócito. A tortuosidade da alça capilar também foi descrita em filhos de mães diabéticas e em adultos descendentes de pacientes com este distúrbio metabólico (25).

Em 1987, Katz \& McNeill atribuíram a tortuosidade da alça à perda da capacidade de regulação metabólica dos esfíncteres pré-capilares em resposta ao acúmulo de catabólitos na região, pois ocorreria um estado permanente de vasodilatação causado pela hipóxia no indivíduo com DM. Este fato causaria aumento da pressão intracapilar e da velocidade do fluxo sangüíneo com conseqüente aparecimento da tortuosidade. Sandeman e cols. (1992) concordaram com essa interpretação, porém correlacionaram-na com o controle da doença, pois observaram-na com maior freqüência em pacientes mal controlados $(24,39)$.

Embora alguns autores não a correlacionassem com a doença, considerando-a como um achado comum no jovem, a tortuosidade da alça capilar foi significativamente mais freqüente nas crianças com DM por nós estudadas $(\mathrm{p}<0,001)$ (tabela 2$)(11,21)$.

A microectasia foi inicialmente descrita por Weiss, em 1916, através da capilaroscopia, quando estudou pacientes diabéticos. Ballantyne \& Loewenstein (1943) mostraram-na como uma dilatação localizada, geralmente, no ramo venoso do capilar $(1,46)$.

Devemos a Ditzel e colaboradores, no entanto, o maior número de trabalhos sobre essa alteração. Em 1954, ele estabeleceu uma correlação entre os achados dos exames realizados na retina com os na prega ungueal que, basicamente, consistiam de congestão, microaneurismas, tortuosidades angulares e edema (11). Já em 1960, associou esta saculação, presente nos ramos capilares e a formação do edema, à congestão venosa (1213). Em trabalhos realizados nos anos seguintes, correlacionou a microectasia à hipóxia, a qual levaria à degeneração do pericito e/ou célula endotelial, favorecendo a sua formação e a neovascularização (14-18).

No nosso trabalho, a microectasia foi observada em $24(80 \%)$ crianças do grupo alvo $(\mathrm{p}<0,001)$ (tabela 2$)$.

A busca de um marcador para a microangiopatia do DM vem sendo tentada pelos pesquisadores em filhos e outros parentes com DM, sendo, freqüentemente, encontradas alterações tanto estruturais quanto metabólicas $(4,11,40)$. Os resultados de nosso trabalho, feito com método não invasivo e de simples execução, sugerem que novos estudos devem ser realizados no sentido de avaliar sua utilidade na detecção precoce das complicações vasculares da doença. 


\section{CONCLUSÃO}

Crianças com DM tiveram, à capilaroscopia da prega ungueal, maior freqüência de alterações da microcirculação do que as normais. Estas alterações foram caracterizadas por espasticidade de alça aferente, dilatação e tortuosidade dos ramos transverso e eferente e presença de microectasias. Estes achados podem ser úteis no diagnóstico da doença e sua aplicação clínica merece ser melhor estudada.

\section{REFERÊNCIAS}

1. Ballantyne AJ, Loewenstein A. Diseases of the retina I. The pathology of diabetic retinopathy. Trans Ophthalmol Soc UK 1943;63:95-115. Apud: McMillan, DE. Deterioration of the microcirculation in Diabetes. Diabetes $1975 ; 24: 944-57$.

2. Banting FG, Best $\mathrm{CH}$, Collip JB, Campbell WR, Fletcher AA. Pancreatic extracts in the treatment of Diabetes mellitus. Can Med Assoc J 1922;12:141-6.

3. Berardinelli W. Semiologia Pervivascular 1939. Tese. Rio de Janeiro. Oficinas Gráficas "Alba", LXI.

4. Bernardini EM. Estudo da Microcirculação em Descendentes de Diabéticos Através da Capilaroscopia 1992. Tese. Escola Paulista de Medicina, São Paulo.

5. Bollinger A, Frey J, Jäger K, Ferrer J, Seglias J, Siegenthaler W. Patterns of diffusion through skin capillaries in patients with long term Diabetes. N Eng J Med 1982;307:1305-10.

6. Bollinger A, Jäger K, Röten A, Timeus C, Mahler F. Diffusion pericapillary distribution and clearence of $\mathrm{Na}$-fluorescein in the human nailfold. Pflüzer Arch 1979;382:137-43.

7. Bollinger A, Frey J, Jäger K, Ferrer J, Seglias J, Siegenthaler W. Patterns of diffusion through skin capillaries in patients with long term Diabetes. N Eng J Med 1982;307:1305-10.

8. Carpentier $\mathrm{P}$, Jeannoel $\mathrm{P}$, Bost $\mathrm{M}$, Franco $\mathrm{A}$. La capillaroscopie péri-unguéale en pratique pédiatrique. Pédiatrie 1988;43:165

9. Chen D, Studer A, Leitölf O, Schaffner T, Mahler F. Increased collagen around deformed finger nailfold capillaries in type I Diabetes mellitus. Klin Wochenschr $1991 ; 69: 336-41$.

10. Cony M, Klene-Boudard C, Sarrat P, Taieb A, Maleville J. Ėtude des aspects capillaroscopiques péri-unguéaux chez lènfant normal. Arch Fr Pediatr 1988:49:171-84.

11. Ditzel J. Morphologic and hemodinamic changes in the smaller blood vessels in Diabetes mellitus I. Considerations based on literature. N Eng J Med 1954;250:541-6.

12. Ditzel J. The in vivo reactions of the small blood vessels to Diabetes mellitus. Acta Med Scand (suppl 476) 1967:123-34.

13. Ditzel J. Functional microangiopathy in Diabetes mellitus. Diabetes 1968;17:388-97.

14. Ditzel J. Caliber changes in the cutaneous microvessels as related to changes in basal metabolism of diabetics. Microvasc Res 1974;8:259 (abstract)
15. Ditzel J, Standl E. The problem of tissue oxygenation in Diabetes mellitus I. Its relation to the early functional changes in the microcirculation of diabetic subjects. Acta Med Scand (suppl 578) 1975:49-58.

16. Ditzel J, Standl E. The problem of tissue oxygenation in Diabetes mellitus II. Evidence of disordered oxygen release from the erytrocytes of diabetics in various conditions of metabolic control. Acta Med Scand (suppl 578) $1975: 59-68$

17. Ditzel J. The problem of tissue oxygenation in Diabetes mellitus III. The "three in one concept" for the development of microangiopathy and a rational approach to its prophylaxis. Acta Med Scand (suppl 578) 1975;69-73.

18. Ditzel J. Affinity hypoxia as a pathogenetic factor of microangiopathy with particular reference to diabetic retinopathy. Acta Endocrinol (suppl 238) 1980;39-55.

19. Dumas R, Desvaux P, Caillard P, Cloarec M. La microangiopathie digitale chez le nourisson. Étude par la capillaroscopie. J Mal Vasc (suppl A) 1987;12:16-20.

20. Farqhuar MG, Hopper J, Moon HD. Diabetic glomerulosclerosis: electron and light microscopic studies. Am J Pathol 1959:35:721-35.

21. Freedlander SO, Lenhart $\mathrm{CH}$. Clinical observation on the capillary circulation Arch Int Med 1922;29:12-32.

22. Gasser P, Berger W. Nailfold videomicroscopy and loca cold test in type I Diabetes. Angiology 1992;43:395-400.

23. Jager E. Beiträge zur pathologie des auges, 1855. Vienna apud McMillan, DE. Deterioration of the microcirculation in Diabetes. Diabetes 1975;24:944-57.

24. Katz MA, McNeill $G$. Defective vasodilatation response to exercise icutaneous precapillary vessels in diabetic humans. Diabetes 1987;36:1386-96.

25. Kiesewetter H, Jung F, Körher W, Wolf S, Kichl R, Reun M, et al. Microcirculation and hemorheology of children with type I Diabetes. Klein Wonchenschr 1986; 1:962-8.

26. Kimmelstiel $\mathrm{P}$, Wilson $\mathrm{C}$. Intercapillary lesions in the glomeruli of the kidney. Am J Pathol 1936; 12:83-105.

27. Landau J, Davis E. The small blood vessels of the conjunctiva and nailfold in Diabetes mellitus. Lancet $1960 ; 1: 731-4$

28. Lombardi WP. The blood pressure in the arterioles, capillaries and small veins in the human skin. Am J Physiol 1912;29:335-62.

29. Lunbaeck K. Long term diabetes. The clinical picture in diabetes mellitus of 15-25 years' duration with a follow up of a regional series of cases. Copenhagen Ejnar Münksgaard 1953, p.138 apud McMillan. The microcirculation in Diabetes Endothelium Lymphatics 1984; 1:3-24.

30. Mayer KM. Observation on the capillaries of the normal infants. Am J Dis Child 1921;22:381-7.

31. McMillan DE. Deterioration of the microcirculation in Diabetes. Diabetes 1975;24:944-57.

32. McMillan DE. The microcirculation in Diabetes. Microcirc Endothelium Lymphatics 1984;1:3-24.

33. Morais IC. Capilaroscopia em Diabéticos 1985. Tese. UFRJ. Rio de Janeiro. 109. 
34. Morais IC, Halfoun VLRC, Fernandes TJ, Fonseca MHGA, Oliveira JEP, Bernardini EM. Capilaroscopia em diabéticos. Arq Bras Endocrinol Metab 1988;5:3-8.

35. Müller $\bigcirc$. Die kapillären der menschlichen korperoberfläsche in Gesunden und Kranken. 1922. Stuttgart. Ferdinand Enke. apud Leader SD. Capillary microscopy in children. Am J Dis 1932;44:403-16.

36. Pazos-Moura CC, Moura EG, Bouskela E, Torres Filho IP, Breitenbach MMD. Nailfold capillaroscopy in Diabetes mellitus: morphological abnormality relationship with microangiopathy. Braz J Med 1987;20:777-80.

37. Priollet $P$, Tubiana Rufi N, Lévy-Maechal C, Czernikon $P$. Microangiopathie digitale des enfants et adolescents diabétiques. Études par capillaroscopie. J Mal Vasc (suppl A) 1987; 12:20-4.

38. Rasio EA, Bendayan M, Goresky CA. Effect of reduced energy metabolism and hyperfusion on the permeability and morphology of the capillaries of an isolates rete mirabile. Circ Res 1989:64:243-54.

39. Sandman DD, Shore AC, Tooke JE. Relation of skin capillary pressure in patients with insulin-dependent Diabetes mellitus to complications and metabolic control. N Eng J Med 1992;327:760-4.

40. Siperstein MD, Norton W, Unger RH, Madison, LL. Muscle capillary basement membrane width in normal, diabetic and prediabetic patients. Trans Ass Am Physicians $1966 ; 79: 330-47$

41. Trevisan G, Rizzi GM, Borzaghini L. Capillaroscopia del vallo ungueale e fluoroangiofrafia retinica nel diabete en età pediatrica. Giorn It Derm Vener 1986;121:169-72.
42. Tooke JE. A capillary pressure disturbance in young diabetics. Diabetes 1980;29:815-9.

43. Tur E, Yosipovitch $G$, Bar-On Y. Skin reactive hyperemia in diabetic patients. A study by laser Dopplerflowmetry. Diabetes Care 1991;14:958-62.

44. Waite JH, Beetham WP. The visual mechanism in the Diabetes mellitus (a comparative study of 2002 diabetics and 457 non-diabetics for control). N Engl J Med 1935 a:212:367-79

45. Waite JH, Beetham WP. The visual mechanism in the Diabetes mellitus. A comparative study of 2002 diabetics and 457 non-diabetics for control). N Engl J Med 1935 b; $212: 429-44$

46. Weiss E. Beobachtung und mikrophotographische darstellung des hautkapillaren am lebenden menschen. Arch Klin Med t. CXIX, 1916 apud Weiss M. La capillaroscopie. Press Med 1921;11:105-7.

47. Zatz R, Brenner BM. Pathogenesis of diabetic microangiopathy. The haemodynamic view. Am J Med 1986;80: 443-53.

\section{Endereço para correspondência:}

Therezinha J. Fernandes

Rua Humberto de Campos, 746/302

22.430-190 Rio de Janeiro, RJ 